\title{
PROJETO E IMPLEMENTAÇÃO DE UMA PLATAFORMA COLABORATIVA PARA AUXILIAR A COMUNIDADE ACADÊMICA COM PROBLEMAS OCASIONADOS PELA PANDEMIA DO COVID-19
}

\author{
Francisco L. de Caldas Filho, Leonardo de Oliveira Almeida, Natalia A. Marques, \\ Paulo H. F. C. Mendes, André Cavalcante, e Rafael T. de Sousa Júnior \\ Departamento de Engenharia Elétrica, Universidade de Brasília (UnB), Brasília-DF, Brasil
}

\begin{abstract}
RESUMO
A pandemia mundial causada pelo vírus COVID-19 trouxe alguns desafios para a humanidade até então inéditos: a necessidade de manter o distanciamento social para reduzir a circulação do vírus sem paralisar diversos setores como comércio e educação. O projeto proposto neste texto tem como objetivo criar uma plataforma de solidariedade permitindo que pessoas pertencentes a grupos de risco possam permanecer em casa enquanto voluntários saudáveis possam realizar algumas tarefas do seu dia a dia. Além desta tarefa, o software desenvolvido neste projeto permite que alunos sejam orientados à distância por professores universitários, mantendo parte das suas tarefas acadêmicas.
\end{abstract}

PALAVRAS-CHAVE

COVID-19, Aplicativo, Rede Solidária, Mobile, Dashboard, IoT

\section{INTRODUÇÃO}

Em Dezembro de 2019, na província de Wuhan, localizada na China, foi identificado um novo tipo de coronavírus. Posteriormente este local viria a ser o epicentro do vírus, desencadeando uma pandemia. Atualmente, segundo dados da universidade Johns Hopkins o COVID-19, este vírus já infectou mais de 20 milhões de pessoas em todo o mundo; um dado atingido em apenas oito meses após seu primeiro caso. De acordo com dados do Ministério da Saúde, no Brasil, em Agosto de 2020, o Covid-19 chegou a infectar mais de 3 milhões de pessoas e ultrapassou a marca de 100 mil mortes. Devido ao cenário caótico no qual o Brasil se encontra, novas ferramentas foram desenvolvidas para amenizar essa situação.

A tecnologia vem influenciando no combate ao novo coronavírus e ajudando na adaptação ao novo estilo de vida de parte da população. Seu uso auxilia a sociedade a lidar com as limitações impostas pela pandemia. Alguns softwares possibilitam, desde o trabalho remoto, compras, ajudando a população a permanecer em isolamento e distanciamento social. Da mesma forma, tem-se a Telemedicina, uma facilidade advinda da tecnologia, através dela pessoas podem consultar-se por meio de ligações telefônicas ou chamadas de vídeo sem a necessidade do deslocamento até o consultório, reduzindo, assim, o risco de contágio. Desse modo, o uso de softwares pode facilitar o dia a dia da população, tanto para o cenário pandêmico, quanto para o cotidiano de forma geral.

Dadas as necessidades gerais da população, incluindo-se as inseridas diante desse novo contexto vivido em 2020, docentes e discentes iniciaram o projeto do aplicativo Amoris - IoT Mobile - com o objetivo de auxiliar alunos e cidadãos, de forma geral, a se adaptarem aos empecilhos impostos pela quarentena.

O aplicativo possui diversas funcionalidades para facilitar o distanciamento social, tais como:

- Voluntariado: os usuários do aplicativo podem se inscrever para usufruir dessa funcionalidade ou para contribuir como um prestador de serviços. Alguns dos serviços que podem ser prestados pelos voluntários são: passear com um animal de estimação, tirar dúvidas do usuário virtualmente relacionadas à sua área de atuação profissional (advogado(a), professor(a), psicólogo(a), entre outros); fazer compras no mercado para cidadãos do grupo de risco, sejam eles idosos, pessoas com 
comorbidades ou doenças crônicas. Pode-se utilizar da ferramenta para prestação de qualquer tipo de atividade voluntária, mesmo as não citadas como exemplos.

- Saúde: o usuário pode informar a uma autoridade de saúde pública se encontra-se com os sintomas relacionados à COVID-19, receber algumas recomendações advindas da Organização Mundial da Saúde (OMS) e, além disso, pode informar se está em emergência médica, necessitando de um atendimento imediato.

- Orientação: o usuário que for estudante ou professor da UnB também pode fazer uso da funcionalidade de orientação. Os alunos podem expressar suas dúvidas acadêmicas conforme o departamento da área em questão. Os professores têm acesso às chamadas dos alunos por meio de uma fila de orientações, toda interação final entre o aluno e o professor é feita via e-mail.

- Pânico: devido a preocupações com possíveis aumento na criminalidade pós pandemia, foi desenvolvida a funcionalidade pânico, pensada para uso exclusivo dentro do campus da universidade. O Campus da UnB possui 4.787.449 metros quadrados, com salas de aula, laboratórios, auditórios e apartamentos para moradia de estudantes. Caso o usuário se sinta em situação de perigo, pode solicitar apoio à equipe de segurança da UnB, utilizando apenas gestos no celular.

Esse projeto pretende promover um acesso mais facilitado aos dados relacionados à Covid-19, além de promover um ambiente intuitivo, trazendo diversas funcionalidades, visando auxiliar os usuários em suas mais diversas necessidades, especialmente durante a pandemia.

Essa aplicação dispõe de mecanismos de segurança, para garantir confidencialidade, integridade e disponibilidade das informações sensíveis, que são solicitadas ao realizar o cadastro para ingressar na aplicação e para se tornar um voluntário.

Este documento está dividido em seis seções, sendo esta a Introdução. A seguir, a seção 2, que visa repassar alguns conceitos e artigos publicados que são relevantes na composição deste projeto, e depois, a seção 3, que contextualiza tecnicamente a metodologia utilizada no projeto. Posteriormente, a seção 4, que apresenta as medições e verificações feitas com a execução do projeto; a seção 5 comenta as dificuldades, possibilidades de extensão e perspectivas futuras; e por fim, a Referências, que lista as referências citadas por todo o trabalho.

\section{ARQUITETURA PROPOSTA (APLICATIVO AMORIS - IOT MOBILE APP)}

O aplicativo Amoris foi desenvolvido com o objetivo de atender às demandas urgentes da população detectadas durante a pandemia, tais como a redução da circulação de idosos e pessoas pertencentes a grupos de risco, unindo, assim, usuários que precisam ficar isolados com voluntários que podem doar o seu tempo e serviços especializados, tais como atendimento médico e psicológico à distância, facilitando a vida de quem necessita ficar em casa ou não tem meios de se locomover. A rede solidária poderá ser mantida até mesmo após o fim da pandemia, facilitando as ações de pessoas que tem interesse em continuar prestando serviços voluntários. A figura 1 descreve os componentes da plataforma, que serão explicados a seguir nesta seção.

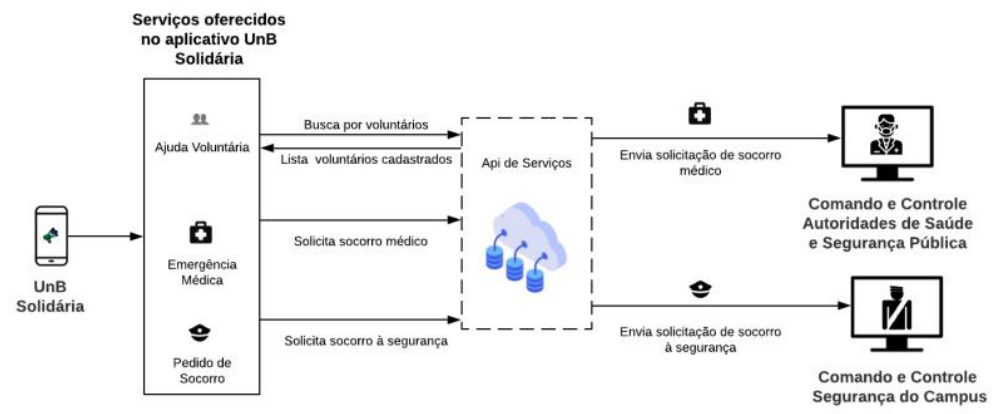

Figura 1. Arquitetura e componentes do software UNB Solidária 


\subsection{Aplicativo Amoris IoT App}

O aplicativo Amoris IoT permite que os usuários tenham acesso a diversos serviços oferecidos pelos voluntários e pela Instituição e é dividido em três principais funcionalidades: ajuda voluntária, emergência médica e pedido de socorro. Cada uma destas funcionalidades será descrita nesta seção. Para os usuários utilizarem o aplicativo, eles precisam realizar um cadastro, informando os seus dados pessoais, concordando com os termos de uso e informando se possuem alguma doença pré-existente que os caracterize como fazendo parte do grupo de risco

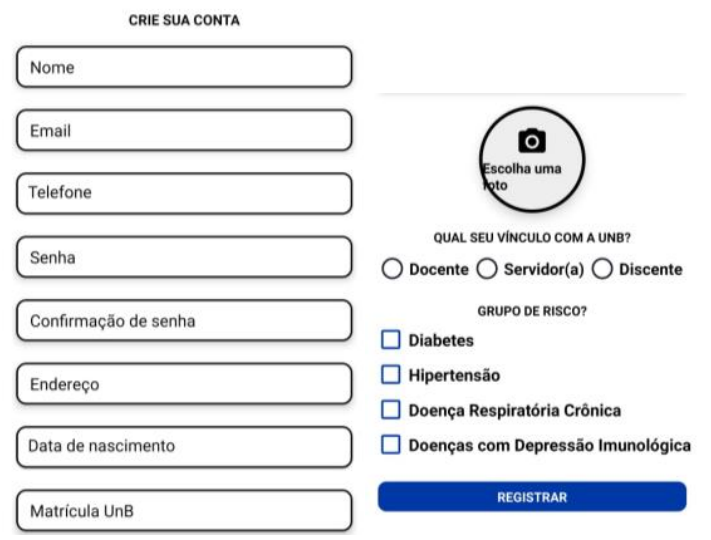

Figura 2. Telas de cadastros

\subsection{Ajuda Voluntária}

A ajuda solidária possui dois atores diferentes: o voluntário, que irá oferecer os seus serviços à comunidade acadêmica, e o usuário que irá utilizar os serviços oferecidos pelos diferentes voluntários. Ao se cadastrar na plataforma, os voluntários definem quais serviços têm disponibilidade de oferecer. Por questões de segurança, são aceitos apenas voluntários que possuam algum vínculo com a Instituição da Universidade de Brasília, permitindo a averiguação dos dados cadastrais. Diversos serviços de voluntariado são permitidos, desde tarefas do quotidiano, tais como realizar compras no supermercado ou consultas por Telemedicina, além de atendimento psicológico. Conforme estudo de Higor Leite and Ian R. Hodgkinson and Thorsten Gruber, grandes períodos de isolamento social podem aumentar níveis de stress e ansiedade, e o atendimento remoto permite que o indivíduo realize a consulta sem sair de casa, reduzindo a quantidade de pessoas que estão circulando na rua.

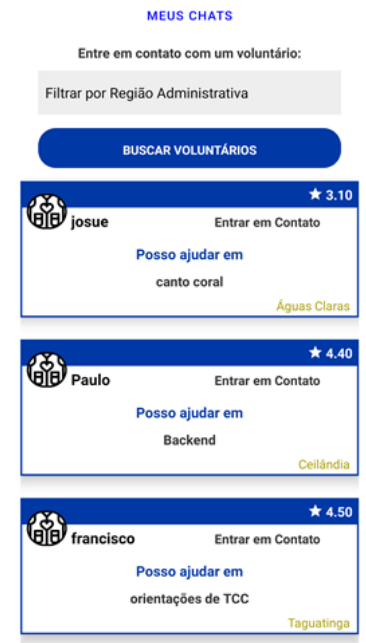

Figura 3. Tela para Solicitação de Ajuda 


\subsection{Emergência Médica}

Visando o pronto atendimento de pessoas que estão apresentando sintomas do COVID-19, o nosso aplicativo desenvolveu uma solução onde o usuário pode informar se está sentindo algum sintoma grave e solicitar auxílio médico emergencial. Ao disparar o pedido de ajuda, este poderá ser encaminhado para uma central de apoio, que possui um painel para visualização e atendimento destes eventos.

Esta funcionalidade permite que equipes de profissionais na área de saúde possam identificar pedidos de socorro, entrando em contato imediatamente com o usuário e visualizando no mapa informações importantes como tempo de deslocamento e distância entre a central e o solicitante da emergência.

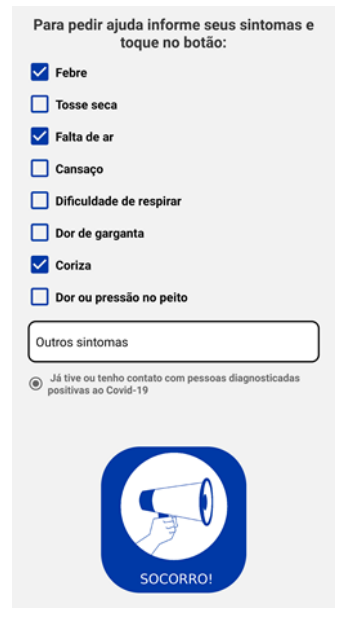

Figura 4. Tela Pedido de Emergência Médica

\subsection{Pedidos de Socorro}

A reitoria da Universidade de Brasília, preocupada com a possibilidade de haver um aumento na criminalidade nas dependências do campus após o retorno das aulas presenciais, solicitou o desenvolvimento desta funcionalidade, com o objetivo de permitir aos usuários solicitar apoio da equipe de segurança da universidade. É sabido que a UnB conta com uma grande área territorial e as rondas realizadas pelos guardas da universidade não são tão efetivas, uma vez que eles não conseguem percorrer todo o campus em um curto período. Sendo assim, essa funcionalidade facilita os agentes de segurança saberem onde devem atuar em emergências como casos de violência no campus ou até mesmo promover apoio para situações menos críticas como acompanhar alunos até a parada de ônibus em horários de pouco movimento. $\mathrm{O}$ aplicativo foi desenvolvido para enviar um alerta para uma central especializada. Este acionamento é feito chacoalhando o celular rapidamente, desta forma, caso o usuário esteja impossibilitado de manusear o aplicativo, a central de segurança poderá receber o alerta e poderá enviar uma equipe até o local para averiguar se houve algum problema.

\subsection{API de Serviços}

O projeto foi desenvolvido utilizando uma arquitetura cliente-servidor, em que o cliente é o dispositivo móvel, onde o aplicativo é instalado, e o servidor é uma API REST, de acordo com Li et al. (2016) com seu banco de dados relacional. Segundo Stonebraker (2010), ambos hospedados em nuvem para oferecer alta disponibilidade para os usuários.

A API, desenvolvida com a plataforma Javascript NodeJS para os autores Tilkov e Vinoski (2010), utiliza o padrão de projeto MVC (Model-View-Controller) abordado por Lucassen e Maes (2011), mais especificamente MC, visto que todas as interfaces, ou Views, estão contidas no aplicativo móvel. A API é responsável pelo fornecimento dos endpoints/rotas a serem consumidos pelo aplicativo, a aplicação das regras de negócio (Controller) e persistência das informações coletadas no banco de dados (Model). Em se tratando dos endpoints existe uma clara separação entre eles de acordo com qual entidade do sistema estão 
relacionados. Para cada uma destas entidades existe uma série de operações padrões invocados por cada endpoint que, em geral, estão relacionadas ao chamado CRUD (CREATE-READ-UPDATE-DELETE), segundo Castillo (2017). As entidades do sistema são:

- Usuário: agrega todas as operações, regras de negócio e dados dos usuários do sistema. Possui cinco endpoints utilizados para cadastro de novos usuários, atualização de dados, leitura da tabela de usuários ou de um usuário específico, login e recuperação de senha.

- Voluntário: é o usuário que se cadastrou como voluntário e passa a aparecer na lista de voluntários ofertada aos usuários que buscam algum tipo de auxílio em atividades variadas. Assim como a entidade Usuário, Voluntário possui endpoints, mais especificamente oito, responsáveis pela criação, leitura e atualização dos dados de voluntário(s). Este último é muito utilizado principalmente nos casos em que o Voluntário declara estar com sintomas de Covid-19, atualizando, assim, seu estado de saúde, ou quando ele simplesmente deseja sair da lista de voluntários. Quatro dos outros endpoints são para criação e atualização das notas e comentários fornecidos pelos usuários para cada voluntário.

- Socorro de Saúde: esta entidade está relacionada aos pedidos de socorro de saúde e tem dois endpoints, um para receber os pedidos de socorro disparados pelos usuários contendo suas informações como nome, número de telefone e posição geográfica (GPS), e outro para consultar esses pedidos e entregá-los à central de comando e controle.

- Socorro de Segurança: Muito semelhante ao socorro de saúde, o socorro de segurança possui dois endpoints, um para receber as solicitações de socorro, e outro para entregá-los à central de comando e controle.

- Orientação Acadêmica: alunos universitários podem solicitar apoio para tirar dúvidas em seu trabalho de conclusão de curso, na resolução de exercícios ou na elaboração de artigos acadêmicos, recebendo suporte dos professores da instituição.

- Central de Comando e Controle: a central desenvolvida neste sistema permite a visualização separada de eventos de saúde pública através dos alertas gerados relacionados a problemas de segurança no Campus. Através do controle de perfis é possível criar visualizações para as equipes que irão atuar no socorro médico e para as equipes que irão visualizar e tratar os eventos de segurança médica.

A visualização dos eventos pode ser feita em um mapa, utilizando a API do Google e permitindo que a equipe de monitoração tenha uma visão precisa de onde a solicitação foi realizada. Ao fazer um pedido de socorro é utilizado a localização fornecida pelo Celular, com uma precisão aproximada de 4,9 metros GPS GOV (2020) permitindo identificar de onde está partindo o pedido de auxílio. Para facilitar o controle, há um painel de eventos onde o usuário poderá visualizar a lista de todos os chamados que recebeu ou analisar qual a tratativa que foi dada.

\subsection{Segurança}

Os mecanismos de segurança utilizados para criptografia das senhas de cadastro são feitos utilizando o algoritmo de hash MD5, que é um hash unidirecional de 128 bits, isso significa que o hash não pode ser convertido para senha. A verificação é feita com a senha inserida pelo usuário e a senha contida no banco de dados. Para realizar a conexão do aplicativo com o banco de dados, temos um gateway de borda que utiliza protocolo https para fazer esse controle de informações.

\section{TESTES E RESULTADOS}

Tabela 1. Cenário de Testes

\begin{tabular}{|c|c|c|c|}
\hline Cenário & Req/Thread & Threads & Total de Reqs em 30s \\
\hline $\mathbf{1}$ & 100 & 12 & 36000 \\
\hline $\mathbf{2}$ & 200 & 12 & 72000 \\
\hline $\mathbf{3}$ & 400 & 12 & 144000 \\
\hline $\mathbf{4}$ & 800 & 12 & 288000 \\
\hline $\mathbf{5}$ & 1000 & 12 & 360000 \\
\hline
\end{tabular}




\section{\$ wrk -t12 -c1000 -d30s https://api.amoris. redes.unb.br}

Figura 5. Exemplo de teste de carga utilizando 12 threads e 1000 requisições

Devido ao tipo de urgência relacionada aos pedidos de socorro que são realizados pelo aplicativo móvel, foram desenvolvidos alguns testes com o intuito de aferir a capacidade da API do aplicativo móvel em lidar com uma grande carga de requisições em curtos períodos de tempo para mostrar que, mesmo em um ambiente onde diversos usuários solicitem ajuda, em curtos espaços de tempo, a API mantém sua robustez e velocidade nas respostas.

Visando comprovar sua capacidade para lidar com uma grande quantidade de requisições, foram realizados cinco testes de carga utilizando a ferramenta WRK de acordo com Gozer (2018), que permite utilizar diferentes combinações de quantidades de threads, tempo de carga e número de requisições, como exemplificado na Figura 5. Em cada cenário de teste, uma determinada quantidade de requisições foi feita por cada um dos 12 threads durante os trinta segundos (30s) de teste para sobrecarregar a API, como pode ser visto na Tabela 1. Ao final de cada teste, foi obtido o relatório de latência média mostrado na Figura 7 e a quantidade de respostas que a API foi capaz de responder em 30 segundos na Figura 6.

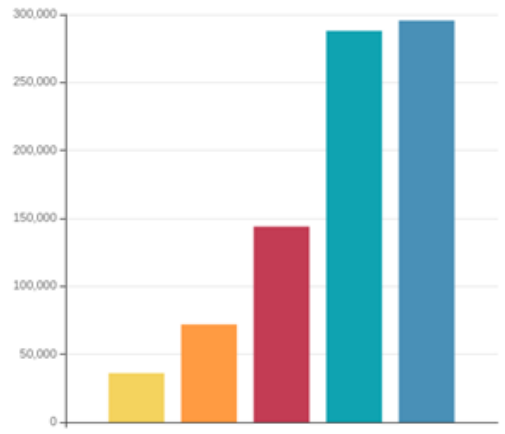

100 reqs 200 reqs 400 reqs 900 reqs 1000 reqs
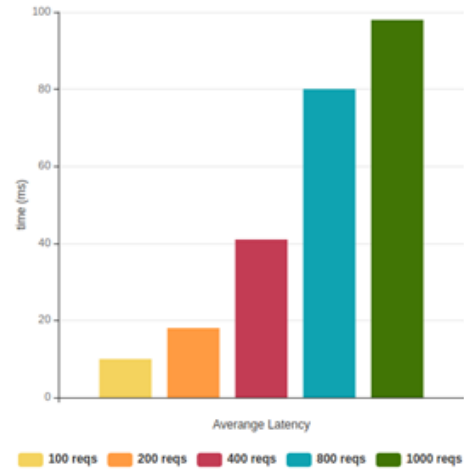

Figura 6

De posse dos dados obtidos nos testes, é possível realizar algumas análises para caracterizar a robustez da API do aplicativo móvel. O primeiro aspecto relevante a ser destacado é que a API conseguiu responder a todas as requisições dos cenários 1 a 4 da Tabela 1, contudo não conseguiu responder ao cenário 5 que fez 360000 requisições e obteve 295655 respostas, que representa retorno para 82,1\% do total das requisições e esse valor demonstrou ser o número máximo de respostas da API no período de 30 segundos. Outro ponto relevante é a latência média que se manteve muito baixa, mesmo no caso extremo do cenário 5 onde não passou dos $100 \mathrm{~ms}$, como mostrado na Figura 7, o que pode ser considerado um número muito baixo dada a quantidade de solicitações atendidas.

As análises apresentadas, baseadas em testes que são muito mais extremos que o cenário real, onde o aplicativo móvel e sua API são utilizados, demonstram que o sistema em si tem total capacidade de atender às solicitações feitas por seus usuários, mesmo em situações de altíssima demanda.

\section{CONCLUSÃO E TRABALHOS FUTUROS}

Focando no auxílio no combate ao Covid-19, o desenvolvimento do aplicativo móvel exposto neste artigo torna-se extremamente interessante para a sociedade, já que com ele é possível realizar solicitações de ajuda médica, solicitação de ajuda voluntária entre os usuários da aplicação, e, para usuários envolvidos com a Universidade de Brasília (UnB), o botão de pânico proporciona uma maior segurança no campus universitário.

Como mostrado nos resultados dos testes de capacidade da API, a aplicação encontra-se preparada para um atendimento satisfatório e eficaz das diversas requisições possíveis no aplicativo, já que mesmo estando em uma simulação onde houve números muito maiores de requisições simuladas do que haveria em casos mais próximos do esperado, apresentou baixa latência na resposta. 
Os bons resultados apresentados pela aplicação motivam a equipe a melhorar ainda mais a solução proposta neste artigo, desenvolvendo novas funcionalidades, corrigindo e aprimorando o código. Além disso, almeja-se aprimorar a segurança do aplicativo, realizando o cadastro com autenticação em duas etapas e realizando o armazenamento das solicitações de auxílio voluntário. Com essas atualizações, haverá melhoras na interface e na experiência, para que o usuário sinta-se confortável e seguro utilizando-se da solução proposta. Por fim, o aplicativo encontra-se disponível na plataforma Google Play Store para download e utilização e entrará em fase de testes com um número maior de usuários. Será necessário também montar as equipes médicas e de segurança que irão realizar o tratamento dos alarmes enviados pelos usuários.

\section{AGRADECIMENTOS}

Os autores agradecem o apoio das Agências Brasileiras de Pesquisa, desenvolvimento e inovação CNPq (Projetos INCT SegCiber 465741/2014-2, PQ-2 312180/2019-5 e LargEWiN BRICS2017-591), CAPES (Projetos FORTE 23038.007604/2014-69 e PROBRAL 88887.144009/2017-00) e FAPDF (Projetos UIoT 0193.001366/2016 e SSDDC 0193. 001365/2016), bem como o suporte do Laboratório LATITUDE/UnB (Projeto SDN 23106. 099441/2016-43), a cooperação com o Ministério da Economia (TEDs DIPLA 005/2016 e ENAP 083/2016), o Gabinete de Segurança Institucional da Presidência da República (TED 002/2017), a Advocacia-Geral da União (TED 697.935/2019) e o Conselho Administrativo de Defesa Econômica (TED 08700.000047/2019-14).

\section{REFERÊNCIAS}

"Boletins epidemiológicos" Ministério da Saúde. [Online]. Available: https://www.saude.gov.br/boletinsepidemiologicos [Ultimo acesso em: 17/08/2020]

Castillo, J. N.; Garcés, J. R.; Navas, M. P.; Segovia, D. F. J. \& Naranjo, J. E. A., 2017. "Base de Datos nosql: Mongodb vs. Cassandra en Operaciones Crud (create, read, update, delete),"Revista Publicando, vol. 4, no. 11,pp. 79-107.

Chand, D.; Nayak, S.; Bhat, K. S.; Parikh S.; Singh, Y. \& Kamath, A. A., “A mobile application for Women's Safety: WoS App.” 2015 IEEE Region 10 Conference TENCON; Macao. 2015 Nov 1-4. pp. 1-5.

“Covid-19 dashboard by the center for systems science and engineering (csse) at Johns Hopkins university (jhu),"Johns Hopkins University.[Online]. Available:_https://coronavirus.jhu.edu/map.html_[Ultimo acesso em:30/07/2020]

Glozer, W., 2018. "wrk-a http benchmarking tool,"

"GPS Accuracy” U.S. government. [Online]. Available: https://www.gps.gov/systems/gps/performance/accuracy/ [Ultimo acesso em: 29/08/2020]

Imran, A.; Posokhova, I.; Qureshi, H. N.; Masood, U.; Riaz, S.; Ali, K.; John, C. N. \& Nabeel, M., 2020. “Ai4covid-19: Ai enabled preliminary diagnosis for covid-19 from cough samples via an app." arXiv preprint arXiv:2004.01275.

Jaffe, E.; Dadon, Z. \& Alpert, E. A., 2018. "Wisdom of the crowd in saving lives: the life guardians app." Prehosp Disaster Med; 35:1-3.

Leite, H.; Hodgkinson, I. R. \& Gruber, T., 2020 "New development: 'Healing at a distance'-telemedicine and COVID-19.” Public Money Management, vol. 40, no. 6, pp. 483-485, DOI: 10.1080/09540962.2020.1748855.

Li, L.; Chou, W.; Zhou, W. \& Luo, M., 2016. "design patterns and extensibility of rest api for networking applications,"IEEE Transactions on Network and Service Management, vol. 13, no. 1, pp. 154-167.

Lucassen, J. M. \& Maes, S. H., "Mvc (model-view-controller) based multi-modal authoring tool and development environment," Mar. 1 2011, uS Patent 7,900,186.

Schinköthe, T.; Gabri, M. R.; Mitterer, M.; Gouveia, P.; Heinemann, V.; Harbeck, N. \& Subklewe, M., "A Web- and App-Based Connected Care Solution for COVID-19 In- and Outpatient Care: Qualitative Study and Application Development." JMIR Public Health Surveill 2020 Jun 01;6(2):e19033.

Stonebraker, M., "Sql databases v.nosqldatabases," Commun.ACM, vol.53, no.4, pp.10-11, Apr.2010. [Online]. Available:https://doi.org/10.1145/1721654.1721659 [Ultimo acesso em: 23/07/2020]

Theocharis, Y.; Vitoratou, S. \& Sajuria, J., 2017. "Civil Society in Times of Crisis: Understanding Collective Action Dynamics in Digitally-enabled Volunteer Vetworks." Journal of Computer-Mediated Communication, pp. 248-265, DOI: $10.1111 /$ jcc4.12194

Tilkov, S. \& S. Vinoski., 2010. "Node.js: Using Javascript to Build High-performance Network Programs," IEEE Internet Computing, vol. 14, no. 6, pp. 80-83. 